\title{
Importância da alimentação saudável no tratamento da depressão em infanto-juvenil
}

\author{
Importance of healthy eating in the treatment of depression in children \\ Importancia de una alimentación saludable en el tratamiento de la depresión infantil
}

Recebido: 22/11/2021 | Revisado: 27/11/2021 | Aceito: 28/11/2021 | Publicado: 04/12/2021

\author{
Márcia Maria Silva da Silveira \\ ORCID: https://orcid.org/0000-0001-7778-0509 \\ Universidade Nilton Lins, Brasil \\ E-mail: msej1.76@gmail.com \\ Erick Frota Gomes Figueiredo \\ ORCID: https://orcid.org/0000-0002-6127-0544 \\ Universidade Nilton Lins, Brasil \\ E-mail: erick.figueiredo@uniniltonlins.edu.br
}

\begin{abstract}
Resumo
Depressão é um termo genérico utilizado para descrever um leque de distúrbios depressivos, tais como depressão major, distimia, doença bipolar. O objetivo do estudo é descrever a importância da alimentação saudável no tratamento da depressão em infanto-juvenil. Através dos objetivos específicos: Relatar as causas da depressão; avaliar o processo da depressão no eixo intestino-cérebro; conhecer os benefícios da alimentação saudável no tratamento da depressão. A metodologia aplicada foi através da revisão bibliográfica, com levantamento de pesquisas através dos bancos de dados disponíveis eletronicamente na BVS (Biblioteca Virtual em Saúde), Scientific Electronic Library Online (SCIELO), Literatura Latino-Americana e do Caribe em Ciências da Saúde (LILACS), utilizou-se também consulta em teses, monografias, dissertações, manuais técnicos e livros. Os resultados indicaram que a alimentação saudável no estado de depressão se torna necessário pois auxilia no controle das crises depressivas regulando os sintomas e equilibrando o sistema nervoso central. Tais resultados denotam a necessidade de realizar mais trabalhos sobre a importância da alimentação na depressão, para melhor compreensão do processo que ocorre no intestino-cérebro.
\end{abstract}

Palavras-chave: Depressão; Alimentação adequada; Qualidade de vida.

\begin{abstract}
Depression is a generic term used to describe a range of depressive disorders such as major depression, dysthymia, bipolar disorder. The aim of the study is to describe the importance of healthy eating in the treatment of depression in children and adolescents. The methodology applied was through bibliographic review, with research survey through databases available electronically in the VHL (Virtual Health Library), Scientific Electronic Library Online (SCIELO), Latin American and Caribbean Literature in Health Sciences (LILACS), consultation in theses, monographs, dissertations, technical manuals and books was also used. The results indicated that healthy eating in a state of depression becomes necessary as it helps to control depressive crises, regulating symptoms and balancing the central nervous system. These results show the need to carry out more work on the importance of nutrition in depression, in order to better understand the process that occurs in the intestine-brain.
\end{abstract}

Keywords: Depression; Adequate food; Quality of life.

\section{Resumen}

La depresión es un término genérico que se utiliza para describir una variedad de trastornos depresivos como depresión mayor, distimia, trastorno bipolar. El objetivo del estudio es describir la importancia de una alimentación saludable en el tratamiento de la depresión en niños y adolescentes. La metodología aplicada fue mediante revisión bibliográfica, con relevamiento de la investigación a través de bases de datos disponibles electrónicamente en la BVS (Biblioteca Virtual en Salud), Biblioteca Científica Electrónica en Línea (SCIELO), Literatura Latinoamericana y del Caribe en Ciencias de la Salud (LILACS), consulta en tesis, monografías, También se utilizaron disertaciones, manuales técnicos y libros. Los resultados indicaron que la alimentación saludable en un estado de depresión se vuelve necesaria ya que ayuda a controlar las crisis depresivas, regulando los síntomas y equilibrando el sistema nervioso central. Estos resultados muestran la necesidad de realizar más trabajos sobre la importancia de la nutrición en la depresión, con el fin de comprender mejor el proceso que ocurre en el intestino-cerebro.

Palabras clave: Depresión; Alimentación adecuada; Calidad de vida.

\section{Introdução}

Atualmente, o número de pessoas no mundo afetadas por doenças mentais é alarmante, visto que, em geral, os 
transtornos são caracterizados por variações de humor, distúrbios de apetite e de sono, baixa autoestima, pensamento acelerado, entre outros (Jorge et al., 2019 p.103).

É caracterizada por alterações no apetite ou peso, diminuição da energia e sono frequente, sentimentos de desvalia ou culpa, dificuldades para pensar, concentrar-se ou tomar decisões, e em casos graves, pensamentos suicidas recorrentes ou tentativas de suicídio. No Brasil, a depressão atinge 11,5 milhões de pessoas, cerca de 5,8\% da população (Saraiva et al, 2020).

O termo Depressão, que deriva do latim depressus, é utilizado para denominar o transtorno mental que tem como característica uma tristeza recorrente e a redução do interesse a atividades variadas, ocasionando uma impossibilidade da realização de atividades diárias durante tempos superiores a duas semanas. Estima-se que mais de 300 milhões de pessoas sofram de depressão (Opas, 2017).

Silva e Loureiro (2017), relatam que a depressão é um dos transtornos mais prevalentes na população em geral e também na universitária.

Uma dieta adequada, que forneça os níveis necessários de minerais, ácidos graxos e vitaminas gera a possibilidade da diminuição dos sintomas depressivos podendo até mesmo inibir as crises depressivas. Dentro dos diversos tipos de dieta existentes a dieta mediterrânea aparece como uma das mais promissoras no tocante ao controle da depressão, que se deve ao fato de ser composta, em grande maioria por alimentos ricos em substâncias benéficas para o quadro promovendo uma melhora da saúde do paciente e sem contraindicações (Silva \& Cardoso, 2021).

O presente estudo tem por objetivo geral descrever a importância da alimentação saudável no tratamento da depressão em infanto-juvenil. Através dos objetivos específicos: Relatar as causas da depressão; avaliar o processo da depressão no eixo intestino-cérebro; conhecer os benefícios da alimentação saudável no tratamento da depressão.

O estudo se justifica pelo índice elevado de depressão no mundo, tornando muitas pessoas doentes mentais. O interesse no tema, se relaciona a um caso de depressão na minha família, pois através da alimentação saudável atribui benefícios para o tratamento da depressão. Dessa forma torna-se de extrema relevância a realização do estudo para conhecer os benefícios da alimentação saudável na saúde mental.

\section{Metodologia}

Foi realizado um estudo de revisão interativa, que para Ynayara e Damasio (2016), é um método de pesquisa utilizado desde 1980, que proporciona a integração de conhecimento e a inclusão da aplicabilidade dos resultados de estudos expressivos na prática e atuação profissional. Os resultados buscam ser úteis, acentuando a importância da pesquisa acadêmica, gerando mudanças e atualizações sobre a prática, facilitando assim, as transformações habituais como consequência da pesquisa.

Neste cenário a revisão integrativa surge como uma metodologia que proporciona e dá suporte a tomada de decisão, além de apontar espaços que precisam ser preenchidos com novos estudos e por tolerar delimitações sucintas, possibilitando investigar cuidadosamente o tema escolhido.

Seguindo este caminho, que permite que pesquisas anteriores sejam reunidas, para que se alcancem os objetivos de forma satisfatória, através do levantamento de literaturas disponíveis existentes e para responder às questões levantadas sobre a depressão, pelo qual, conclusões possam ser formadas a partir da análise criteriosa dos resultados de busca e identificação das evidências científicas.

Diante disso, foi realizado um levantamento de pesquisas através dos bancos de dados disponíveis eletronicamente na BVS (Biblioteca Virtual em Saúde), Scientific Electronic Library Online (SCIELO), Literatura Latino-Americana e do Caribe em Ciências da Saúde (LILACS), utilizou-se também consulta em teses, monografias, dissertações, manuais técnicos e livros. 


\section{Resultados e Discussão}

Depressão é um termo genérico utilizado para descrever um leque de distúrbios depressivos, tais como depressão major, distimia, doença bipolar, transtorno afetivo sazonal e depressão pós-parto (Silva, 2019).

Os transtornos depressivos afetam pessoas de todas as idades e a incidência vai ficando maior a cada dia. Esses transtornos apresentam sintomas que abrangem não só o psicológico, mas também aspectos comportamentais, fisiológicos, sociais e econômicos, e os exemplos maiores são a perda de apetite, humor deprimido, insônia, sentimento de culpa excessiva, de inutilidade, baixa autoestima e também retardo psicomotor (Schwan et al., 2011).

Nas crianças o retraimento social, a falta de interesse em atividades cotidianas, a baixa autoestima, a irritabilidade, entre outros, são exemplos de sintomas que elas podem apresentar quando estão com depressão (Carbonara et al., 2012).

Caracteriza-se por humor triste, vazio ou irritável, variações nítidas no afeto, relacionadas a alterações somáticas, cognitiva se neurovegetativas, que comprometem significativamente a capacidade do indivíduo em realizaras atividades cotidianas, com diferentes aspectos de duração, momento ou etiologia presumida (APA DSM-5, 2014).

A depressão é resultado de uma complexa interação de fatores sociais, psicológicos e biológicos. Pessoas que passaram por eventos adversos durante a vida (desemprego, luto, trauma psicológico) são mais propensas a desenvolver depressão. A depressão pode, por sua vez, levar a mais estresse e disfunção e piorar a situação de vida da pessoa afetada e o transtorno em si (Opas, 2017).

Categoricamente, a depressão pode se apresentar de três formas: depressão menor, que caracteriza-se pelo aparecimento de dois ou mais sintomas, por um período de duas semanas ou mais; distimia, que representa o quadro onde os indivíduos exibem três ou quatro sintomas, por no mínimo, dois anos; e ainda a depressão maior, onde observa-se a presença de um episódio depressivo envolvendo sintomas clássicos como humor deprimido, energia diminuída, perda do interesse e anedonia (Gonçalves, et al., 2017 \& Who, 2017).

Segundo Pimenta (2018), os neurotransmissores serotonina, noradrenalina e dopamina desempenham um papel crítico na comunicação neural, influenciando desde movimentos involuntários até o aprendizado e o humor. Esse sistema é complexo e altamente interconectado, podendo seu desequilíbrio gerar a depressão.

Beck e Alford (2016), relatam um contraste do termo depressão como uma imagem que a pessoa deprimida tem de si mesma e os fatos objetivos. Uma mulher rica lamenta-se por não ter recursos financeiros para alimentar seus filhos. Um ator de cinema amplamente reconhecido implora por uma cirurgia plástica por acreditar-se feio. Apesar do sofrimento vivenciado em decorrência dessas ideias autodepreciativas, os pacientes não são facilmente demovidos por evidencias objetivas ou por demonstrações lógicas da natureza insensata desses pensamentos. A depressão tem causado mais sofrimento humano do que qualquer outra das doenças que afetam a humanidade. A condição que hoje rotulamos de depressão foi descrita por alguns autores antigos sob a categoria de "melancolia".

Cavalcante, Minayo e Mangas (2013) relatam que depressão costuma estar associada a algum tipo de perda na habilidade física, aparência, papel social, morte de outros, segurança financeira. Adultos depressivos sentem como se seu mundo tivesse se estreitado, suas escolhas ficassem menores, seus interesses e preferências menos disponíveis. Eles sentem uma tristeza insuportável.

A depressão pode ser desencadeada por fatores biológicos, sendo a genética um fator significativo no desenvolvimento de um quadro depressivo. Além disso, fatores psicológicos causam perda da autonomia e agravamento de quadros patológicos preexistentes no idoso, assim como os fatores sociais que interferem na capacidade funcional, do autocuidado e nas suas relações sociais (Nóbrega et al., 2015).

A depressão além de constituir-se em um sério problema de saúde, é também considerada um forte fator de risco para outros agravos a saúde, como o uso/abuso de álcool e outras drogas, vindo a desenvolver abuso e dependência. Torna-se oportuno 
salientar que também existem os casos onde a pessoa começa a usar drogas e a partir daí desenvolve transtorno psiquiátrico, ou em detrimento da predisposição genética, fator comum que pode contribuir para o desenvolvimento destas duas comorbidades (Cantão et al., 2015).

Borges e Dalmolin (2012) relatam que as causas de depressão podem variar desde fatores psicossociais, como condições adversas que podem influenciar o início e a persistência dos episódios depressivos, fatores genéticos e biológicos, sendo frequentemente atribuída a acontecimentos estressantes e negativo.

De acordo com Camargo et al., (2019) as pressões que ocorrem na fase da adolescência, é crucial para que esses jovens tenham uma maior propensão para o suicídio, por muitas vezes não saberem lidar com as adversidades e nem terem o apoio desejado para encarar essas situações. Apesar das pesquisas apresentarem que as mulheres possuem maior ideação suicida que os homens, a efetividade desse pensamento ocorre mais entre o sexo masculino. A proporção é de quatro homens pra cada mulher que comete suicídio

Antunes et al., (2016) relatam que o indivíduo afetado pela depressão se percebe diferente e se sente discriminado não só por médicos, familiares e amigos como pela sociedade em geral. Assim, os pais também precisam de uma ajuda psicológica para que tenha uma visão mais realista em relação ao seu filho, para que assim ele perceba a situação e trate desde o início.

Como outro fator predisponente para a depressão na criança e no adolescente, as altas taxas de insatisfação corporal podem ser esclarecidas pelo processo a partir do qual as pessoas passam a seguir padrões inadequados de alimentação e atividade física, contribuindo assim para o aumento do peso e consequente insatisfação com o corpo. Para as mulheres, a vontade de melhorar sua imagem e deixar de ser alvo da sociedade por estar fora dos padrões são as principais motivações que levam à busca pela mudança em seus corpos, normalmente querendo ser/parecer mais magra. Entre os homens, esta insatisfação pode ser explicada pela pressão que propõem o padrão de corpo musculoso e atlético (Camargo et al., 2019). eixo microbiota-intestinocérebro é um conjunto de vias neurais e gânglios, que vão envolver diversos sistemas, o Sistema Nervoso Central (SNC), o Sistema Nervoso Entérico (SNE), o Sistema Nervoso Autônomo (SNA), o de vias neurais aferentes e eferentes, sistema imune e sistema endócrino, com o objetivo de relacionar os controles cognitivos e emocionais do cérebro com os gânglios do SNE, assim, o funcionamento de um irá depender em conjunto com o outro, sendo um eixo de sinalização bidirecional (Zorzo, 2017).

Existem ainda evidências que mostram que a microbiota também pode desempenhar um papel importante para o início de patogêneses, através de mecanismos como inflamação e liberação de toxinas, por metabólitos e neuro-hormônios produzidos pelos microrganismos presentes no intestino, onde na ausência de microbiota intestinal saudável, o cérebro não se desenvolve normalmente (Alam, Abdolmaleky \& Zhou, 2017).

A microbiota intestinal consiste no conjunto de microrganismos que residem no intestino humano, encontrando-se em comunicação com o Sistema Nervoso Central através do eixo intestino-cérebro. Essa comunicação é bidirecional, em que um pode influenciar na atividade do outro, por meio da interação entre sinapses do Sistema Nervoso Entérico com o Sistema Nervoso Central, através do nervo vago (Landeiro, 2016).

Essa comunicação integra as sinalizações neural, hormonal e imunológica entre a microbiota e os seus metabólitos com o cérebro, permitindo que este influencie nas funções gastrintestinais e imunológicas (Marese et al., 2019).

O processo de colonização microbiana inicia mecanismos de sinalização que afetam os circuitos neuronais envolvidos no controle motor e no comportamento da ansiedade. Muitos indivíduos que relatam distúrbios no domínio emocional, sendo o mais frequente a ansiedade, nos distúrbios somáticos funcionais apresentam alterações na microbiota intestinal (França et al., 2021).

O predomínio de microrganismos patogênicos, devido ao desequilíbrio da flora intestinal, aumenta a liberação de toxinas, a inflamação e a permeabilidade do epitélio intestinal, o que ativa o sistema nervoso parassimpático e os neurônios aferentes da medula espinal, os quais são responsáveis por modular o sistema nervoso central (SNC) e o sistema nervoso entérico 
(SNE), regulando as características epiteliais intestinais (Landeiro,2016).

Para França et al., (2021), modulação intestinal por meio da dieta ingerida promove um efeito positivo sobre a regulação do estado de humor, tanto em estudos com ratos e humanos. Tais resultados denotam que os probióticos são seguros e que podem ser moduladores do SNC, influenciando positivamente transtornos emocionais. Assim, possuem propriedades antidepressivas e ansiolíticas, principalmente os gêneros Lactobacillus e Bifidobacterium.

Existe um eixo intestino-cérebro que deve ser levado em consideração para tratamento e prevenção de doenças psiquiátricas, tendo mais uma opção de prognóstico, com uso de probióticos, psicobióticos e uma mudança na dieta, como forma de evitar e tratar disbioses e, consequentemente, problemas no sistema nervoso central (Juppa, 2021).

Figura 1: Desenvolvimento paralelo do cérebro e da microbiota intestinal nos primeiros três anos de vida.

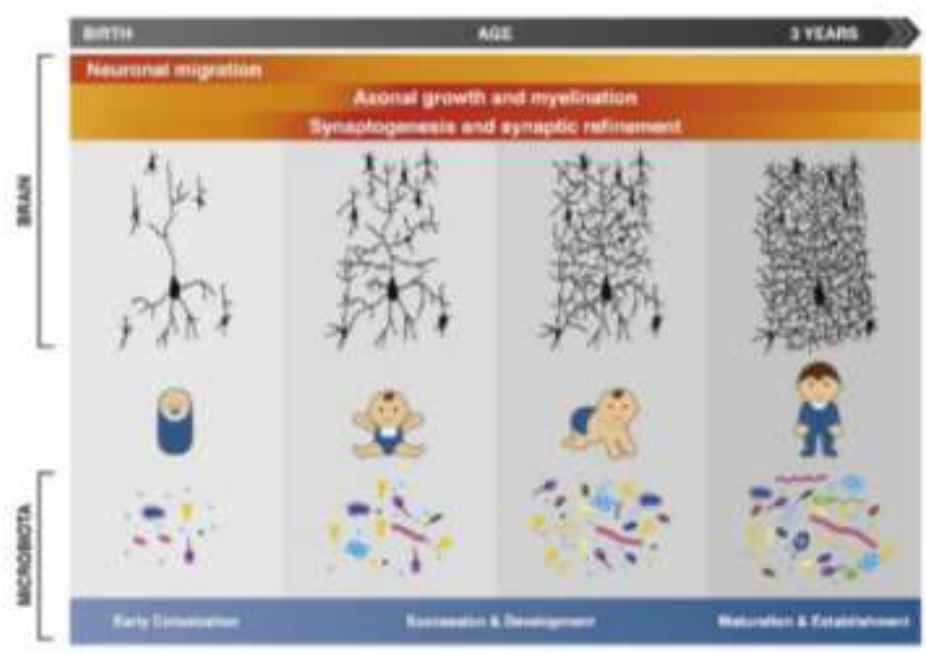

Fonte: Silva (2019).

Silva (2019), relata que o neuro-desenvolvimento se inicia com a migração neuronal, seguida do crescimento axonal e mielinização, assim como da sinaptogénese e o aperfeiçoamento sináptico, de maneira a formar uma rede neuronal de um cérebro inteiramente funcional. Paralelamente decorre um aumento da complexidade da microbiota intestinal até se atingir um microbioma estável, típico de um adulto conforme a Figura 1.

Silva (2019), relata que a exposição a diferentes microrganismos, dietas, stress materno, antibióticos e outros fatores ambientais, principalmente na fase mais precoce da vida, são condições fundamentais para moldar e definir a composição bacteriana e, pelo facto de influenciarem a comunicação com o sistema nervoso, podem contribuir para a etiologia de vários distúrbios do desenvolvimento cerebral.

O intestino possui um sistema de neurônios que é capaz de produzir uma quantidade significativa de serotonina, neurotransmissor que proporciona sensação de bem-estar, exercendo grande influência na qualidade de humor. Sendo assim, verificou-se que existe uma conexão bilateral entre o intestino e o Sistema Nervoso Central (SNC), conhecida como eixo intestino-cérebro, de modo que um influencia o outro (Jorge et al., 2019 p.106).

Existe uma inter-relação intensa entre a microbiota, o eixo intestino-cérebro e as patologias mentais, de modo que, criase, portanto, uma nova perspectiva acerca da fisiopatogenia, prevenção e tratamento da depressão, com a modulação da microbiota intestinal. A modulação do eixo do cérebro -intestino, ainda que não de forma exclusiva, está sendo vista como um alvo atraente para o desenvolvimento de novos tratamentos para uma variedade de distúrbios gastrintestinais e psiquiátricos (Marese et al., 2019).

A terapia nutricional é uma alternativa viável para uma saúde mental de qualidade e se faz relevante devido ao seu custo 
acessível e por não ser invasiva. Portanto, levando-se em consideração a importância de tal temática e a existência de pesquisas ainda muito recentes, é premente a necessidade de se prosseguir com estudos relacionados à alimentação e sua influência na saúde mental (Jorge et al., 2019 p.106).

Alves \& Cunha (2020) relata que é preciso ver a alimentação como forma de garantir melhor qualidade de vida, e já que comer é um direito de todos, torna-se ato político, por isso não precisa e nem deve ser complexo. Além disso, oferecer alimentos saudáveis no lugar de alimentos industrializados e processados é zelar também pela saúde.

A alimentação corresponde a um poderoso aspecto da saúde humana. A má alimentação ou um hábito alimentar equivocado vai impactar sobremaneira no desenvolvimento global do indivíduo (Alves \& Cunha, 2020).

Assim a intervenção nutricional na prevenção e/ou redução da intensidade das crises surge levantando a possibilidade da utilização de uma dieta adequada e balanceada como um grande aliado nesta luta (Senra, 2017).

A Dieta Mediterrânica (DM) é um estilo de vida característico dos países banhados pelo Mar Mediterrânico, tendo como atributos o elevado consumo de alimentos de origem vegetal (nomeadamente cereais pouco refinados, hortofrutícolas, leguminosas e frutos oleaginosos), consumo frequente de pescado, consumo reduzido de produtos açucarados, carnes processadas e carnes vermelhas, uso do azeite como principal fonte de gordura, água como bebida de eleição, preferência por produtos minimamente processados, confecções culinárias protetoras das características nutricionais dos alimentos e promoção da convivência com a família e amigos à hora da refeição (Senra, 2017).

Com a ingestão de alimentos considerados inflamatórios (café preto, mate, chocolate, carnes vermelhas defumadas, chili, carne bovina, tempero pronto Ajinomoto, leite com lactose e doces em geral) ocorre um aumento de estresse devido a incapacidade de metabolização de excessos de gorduras saturadas tais como açúcares e frituras. Quando ocorre a ingestão de alimentos ricos em fibras, minerais, vitaminas e polifenóis consequentemente ocorre a redução dessa inflamação (Dilly et al., 2020).

Silva (2021) relata que para pacientes em tratamento depressivo a atenção nutricional complementar, com inclusão de determinados nutrientes pode apresentar papel fundamental na gênese da depressão, tendo ainda como fator positivo a não existência de efeitos colaterais, o que propicia uma melhora global na saúde do indivíduo.

Ferriani (2021) relata que a vitamina D e o ômega 3 são os nutrientes mais estudados, cuja associação com a depressão já está bem comprovada. Recomenda-se a suplementação com ômega 3 para prevenção e tratamento, assim como a adoção da dieta mediterrânea, aumento no consumo de frutas, vegetais, legumes, cereais integrais, nozes e sementes, e redução da ingestão de alimentos processados, produtos de panificação comercial e doces.

Uma revisão da literatura foi feita acerca do impacto do metabolismo, dieta e microbiota na neurodegeneração, e como esses podem afetar vários aspectos da saúde no que diz respeito ao metabolismo energético, sistema imunológico e a função neuronal. Foi destacado que o sistema nervoso central, que está envolvido na etiologia da depressão, é um órgão altamente demandante de energia, além dos neurônios, que também dependem fortemente da glicose como o principal substrato. Além disso, em condições estressantes, outros recursos, como corpos cetônicos e lactato, fornecidos pelas células, podem ser usados (Gentile et al., 2020). 
Quadro 1: Nutrientes que auxiliam no tratamento da depressão.

\begin{tabular}{|c|c|c|}
\hline Nutrientes & Benefícios & Alimentos \\
\hline Magnésio & $\begin{array}{l}\text { O magnésio desenvolve um importante papel } \\
\text { na regulação iônica cerebral pois tem } \\
\text { participação ativa na formação e utilização de } \\
\text { adenosina trifosfato (ATP). }\end{array}$ & $\begin{array}{ll} & \text { Quiabo; } \\
\text { - } & \text { Banana; } \\
\text { - } & \text { Beterraba; } \\
\text { - } & \text { Abacate; } \\
\text { - } & \text { Amêndoas; e } \\
\text { - } & \text { Nozes. } \\
\end{array}$ \\
\hline Zinco & $\begin{array}{l}\text { Melhorar a sobrevivência das células no SNC } \\
\text { bem como sua importante atuação no sistema } \\
\text { imunológico, reduzindo inflamações, que estão } \\
\text { associadas ao sintoma depressivo, atuando } \\
\text { assim como um agente antidepressivo. }\end{array}$ & $\begin{array}{l}\text { - } \quad \text { Carne vermelha; } \\
\text { - } \quad \text { Leite e derivados; } \\
\text { - } \quad \text { Feijão; } \\
\text { - } \quad \text { Castanha de caju; } \\
\text { - } \quad \text { Amêndoas. } \\
\end{array}$ \\
\hline Triptofano (aminoácido) & $\begin{array}{l}\text { Ajuda a sintetizar serotonina, conhecida como } \\
\text { o "hormônio do prazer", melatonina e niacina. }\end{array}$ & $\begin{array}{ll}\text { - } & \text { Arroz integral; } \\
\text { - } & \text { Feijão; } \\
\text { - } & \text { Carne bovina; } \\
\text { - } & \text { Peixe; } \\
\text { - } & \text { Aves; } \\
\text { - } & \text { Abóbora; } \\
\text { - } & \text { Banana } \\
\text { - } & \text { Manga. }\end{array}$ \\
\hline $\begin{array}{l}\text { Ácidos Graxos (lipídios } \\
\text { compostos). Possuem três tipos: } \\
\text { Ácidos graxos insaturados (AGI), } \\
\text { os ácidos graxos saturados (AGS) } \\
\text { e os ácidos graxos trans (AGT) }\end{array}$ & $\begin{array}{l}\text { Sendo o primeiro o mais indicado para } \\
\text { consumo rico pois o AGS e o AGT está } \\
\text { diretamente ligado ao surgimento de } \\
\text { hipercolesterolemia, podendo alterar as } \\
\text { funções vasculares e plaquetárias, } \\
\text { intensificando as chances do desenvolvimento } \\
\text { de problemas cardiovasculares. Gera melhoras } \\
\text { no humor de pacientes com quadros } \\
\text { depressivos, devido à redução de processos } \\
\text { inflamatórios que afetam negativamente o SNC } \\
\text { e seus neurotransmissores. }\end{array}$ & $\begin{array}{l}\text { - Poli-insaturados: Presentes em castanhas, } \\
\text { sementes e óleos vegetais. Castanhas, sementes } \\
\text { e óleos vegetais. } \\
\text { - Poli-insaturados, presente em peixes. }\end{array}$ \\
\hline Vitamina B6, B9 e B12 & $\begin{array}{l}\text { São relevantes na via metabólica estando } \\
\text { envolvidas na síntese de neurotransmissores do } \\
\text { SNC, tendo participação no metabolismo da } \\
\text { homocisteína (proteína que em altas } \\
\text { concentrações aumenta significativamente a } \\
\text { oxidação por radicais livres). }\end{array}$ & $\begin{array}{ll}\text { - } & \text { Leguminosas; } \\
\text { - } & \text { Hortaliças; } \\
\text { - } & \text { Frutas }\end{array}$ \\
\hline Vitamina D & $\begin{array}{c}\text { A metabolização desta vitamina ocorre no } \\
\text { fígado e por conseguinte nos rins, realizando } \\
\text { sua conversão para uma forma biológica ativa } \\
\text { 1,25-hidroxicalciferol (calcitriol), na qual é de } \\
\text { suma importância na manutenção da } \\
\text { homeostase do cálcio, como também para à } \\
\text { saúde dos ossos }\end{array}$ & $\begin{array}{ll}\text { - } & \text { Salmão; } \\
\text { - } & \text { Atum; } \\
\text { - } & \text { Sardinha; } \\
\text { - } & \text { Cavala; } \\
\text { - } & \text { Gema de ovo; } \\
\text { - } & \text { óleo de fígado de bacalhau. }\end{array}$ \\
\hline
\end{tabular}

Fontes: Baldoino et al. (2021), Silva e Cardoso (2021).

Um consumo alimentar qualitativamente adequado pode contribuir para a prevenção e tratamento do transtorno depressivo maior. O papel da dieta mediterrânea, do ômega 3 e da vitamina D na prevenção e tratamento já está bem estabelecido (Ferriani, 2021).

\section{Conclusão}

Através da alimentação saudável é possível intervir no estado de depressão, tendo seus devidos nutrientes. A partir do compromisso feito pelos pais com a linhagem do infanto-juvenil através de nutrientes necessários como: o magnésio que efetua um papel importante na regulação iônica do cérebro; o zinco que trabalha no sistema imunológico; o triptofano que regula a serotonina e os Ácidos graxos que possuem potencial para reduzir processos inflamatórios. Uma dieta adequada, produz a diminuição dos sintomas depressivos podendo até mesmo inibir as crises depressivas.

Portanto através do presente estudo, os resultados indicaram que a alimentação saudável no estado de depressão se torna necessário pois auxilia no controle das crises depressivas regulando os sintomas e equilibrando o sistema nervoso central.

Tais resultados denotam a necessidade de realizar mais trabalhos sobre a importância da alimentação na depressão, para 
melhor compreensão do processo que ocorre no intestino-cérebro.

\section{Referências}

Alves, G. M. \& Cunha, T. C. O. (2020). A importância da alimentação saudável para o desenvolvimento humano. Perspectivas Online: Humanas \& Sociais Aplicadas, 10(27), 46-62. https://ojs3.perspectivasonline.com.br/humanas_sociais_e_aplicadas/article/view/1966/1661

Alam, R., Abdolmaleky, H. M. \& Zhou, J. (2017) Microbiome, inflammation, epigenetic alterations, and mental diseases. American Journal of Medical Genetics Part B: Neuropsychiatric Genetics, 174(6), 651 - 660, https://pubmed.ncbi.nlm.nih.gov/28691768/

American psychiatric association (APA). (2014). DSM-5: Manual diagnóstico e estatístico de transtornos mentais. Artmed Editora.

Baldoino, F. R. R., Mota G. A, Myrella, C., Cunha, M, Garcês, T. C. C. S. \& Andrade, A. R. O. (2021). Atualizações científicas sobre a deficiência de vitamina D, de ácido fólico e magnésio na depressão. Research, Society and Development, 10(2). http://dx.doi.org/10.33448/rsd-v10i2.12572

Beck, A. T., \& Alford, B. A. (2016). Depressão: causas e tratamento. Artmed Editora.

Bolsoni-Silva, A. T. \&Loureiro, S. R. (2017). O impacto das habilidades sociais para a depressão em estudantes universitários. Psicologia: teoria e pesquisa, 32(4), 1-8. http://dx.doi.org/10.1590/0102.3772e324212

Borges, D. T. \& Dalmolin, B. M. (2012). Depressão em idosos de uma comunidade assistida pela Estratégia de Saúde da Família em Passo Fundo, RS. Revista Brasileira de Medicina de Família e Comunidade, 7(23), 75-82. https://rbmfc.org.br/rbmfc/article/view/381/490

Carbonara, A., Silva, A. F, Maximiano, C. R. \& Silva, D. G. (2012). Depressão infantil: estudo com psicólogos de um centro de atenção psicossocial infantojuvenil. Revista Estudos, 16(16), 229-257. http://ojs.unimar.br/index.php/estudos/article/view/854/501

Cavalcante, F. G., Minayo, M. C. S. \& Mangas, R. M. N. (2013). Diferentes faces da depressão no suicídio em idosos. Ciência \& Saúde Coletiva, 18, 29852994. https://www.scielo.br/j/csc/a/tFcTC5pWsLrHpBhXWZ9FwyB/?format=pdf\&lang=pt

Cantão, L., Fonseca, L. L. K., Silva, T. I. M., Oliveira, M., Oliveira, V. C., Machado, R. M. (2015). Perfil sociodemográfico e clínico de idosos com depressão e o uso de substâncias psicoativas. Revista da Rede de Enfermagem do Nordeste, 16 (3): 355-62.

Camargo, G. A., Ala, G. R., Pina, G. C., Teixeira, L. S \& Luciana, A. J. (2019). Causas de depressão em crianças e adolescentes. RESU -Revista Educação em Saúde, V7(1). http://periodicos.unievangelica.edu.br/index.php/educacaoemsaude/article/view/3805/2643

Dilly, R. S. \& Barretta, C. (2019). Nutricional status and consumpition of inflammatory and anti-inflamatory foods by patients with inflammatory bowel diseases. Journal of Coloproctology, Itajaí, october, 40(2): 99-104. https://rsdjournal.org/index.php/rsd/article/view/21446/18945

França, T. B., Silva, P. F. O. A. \& Santos, N. F. E. (2021). Efeitos de probióticos sobre o eixo microbiota-intestino-cérebro e o transtorno de ansiedade e depressão. Brazilian Journal of Development, 7(2), 16212-16225, 2021. https://www.brazilianjournals.com/index.php/BRJD/article/view/24802/19778

Ferriani, Lara Onofre. (2021). Consumo alimentar e depressão: evidências atuais. Consumo alimentar e depressão: evidências atuais. Ponta Grossa - PR: Atena, Monografia, p. 1-388-416,

Gentile, F., Doneddu, P. E., Riva, N., Nobile-Orazio, E. \& Quattrini, A. (2020). Diet, Microbiota and Brain Health: Unraveling the Network Intersecting Metabolism and Neurodegeneration. Int J Mol Sci, 21(20), 7471. https://pubmed.ncbi.nlm.nih.gov/33050475/

Gonçalves, A. M. C. (2018). Prevalência de depressão e fatores associados em mulheres atendidas pela Estratégia de Saúde da Família. Jornal Brasileiro de Psiquiatria, 67 (2), 101-109.

Jorge, A. K. B., Pacheco, A. F. C. \& Moreira, R. V. (2020). Psiquiatria nutricional: a influência da alimentação na saúde mental. Principais transtornos psíquicos na contemporaneidade, 2, 103. http://brasilmulticultural.org/wp-content/uploads/2020/05/ebook-Principais-transtornos-psiquicos_V-2.pdf

Juppa, L. (2021). Microbiota intestinal e sua influência na depressão. UNISUL - Campus Ilha, Tese doutorado.

Landeiro, J. A. V. R. (2016). Impacto da microbiota intestinal na saúde mental. Dissertação de mestrado integrado em Ciências Farmacêuticas. Instituto Superior de Ciências da Saúde Egas Muniz. https://comum.rcaap.pt/bitstream/10400.26/17565/1/Landeiro_Joana_Almeida_Vil\%c3\%a3o_Raposo.pdf

Ynayara, J. D. V. \& Damasio, L. R. (2016). A importância do Enfermeiro no processo de gerenciamento na Central de Material e Esterilização Hospitalar. Faculdade Integrada De Pernambuco - Facipe, Recife.

Marese, A. C. M., et al. (2019). Principais mecanismos que correlacionam a microbiota intestinal com a patogênese da depressão. Fag Journal Of Health (FJH), 1(3) 232-239

Nóbrega, I. R. A. P., Leal, M. C. C., Marques, A. P. O. \& Vieira, J. C. M. (2015). Fatores associados à depressão em idosos institucionalizados: revisão integrativa. Saúde em Debate, 39(3): 536-550. https://www.scielo.br/j/sdeb/a/gfFFTzQKvvCLzr3SWHCXJ6C/?format=pdf\&lang=pt

OPAS - Organização Pan-americana da Saúde (2017). Depressão: o que você

https://www.paho.org/bra/index.php?option=com_content\&view=article\&id=5372:depressaoo-que-voce-

precisasaber\&Itemid=822\#: : text=A\%20depressão\%20é\%20um\%20transtorno,durante\%20pelo\%20 menos\%20duas\%20semanas .

Pimenta, T. (2018). Conheça todos os tipos de neurotransmissores e saiba porque eles são importantes para sua saúde. Tese doutorado. https://www.vittude.com/blog/neurotransmissores/ 
Research, Society and Development, v. 10, n. 16, e150101623740, 2021

(CC BY 4.0) | ISSN 2525-3409 | DOI: http://dx.doi.org/10.33448/rsd-v10i16.23740

Saraiva, F. R. S.; Carvalho, L. M. F.; \& Landim, L. A. S. R. (2020). Depressão e disbiose. Nutrição Brasil, $18(3)$, 175 - 181. https://www.portalatlanticaeditora.com.br/index.php/nutricaobrasil/article/view/3522/5982

Silva, L. A. \& Cardoso, H. C. S (2021). A influência da alimentação adequada na depressão. Centro Universitário UMA. Dissertação de mestrado. https://repositorio.animaeducacao.com.br/bitstream/ANIMA/17284/1/A\%20INFLU\%C3\%8ANCIA\%20DA\%20ALIMENTA\%C3\%87\%C3\%83O\%20ADEQ UADA\%20NA\%20DEPRESS\%C3\%83O.pdf

Senra, I. C. R. (2017). Alimentação e depressão. Faculdade de Ciências da Alimentação e Nutrição. Universidade do Porto, Tese de Doutorado.

Schwan, S., \& Ramires, V. (2011). Depressão em crianças: uma breve revisão de literatura. Psicol Argum, 28(67), 457-68.

Silva, C. C. L. (2019). Depressão e interconexão microbioma-intestino-cérebro. Tese de Doutorado. Universidade de Coimbra.

Zorzo, R. A. Impacto do microbioma intestinal no Eixo Cérebro-Intestino. International Journal of Nutrology, 10(1), $298 \mathrm{~S}$ - 305S. Edição de março 2017. http://dx.doi.org/10.1055/s-0040-1705652 